\title{
Alexander Subbase Theorem for Filters
}

\author{
by \\ Iwo LABUDA \\ Presented by Czestaw BESSAGA
}

Summary. The theorem in the title is proven. Applications to product theorems are given.

We adopt the terminology of [17], and so a topological space $X=(X, \mathcal{O})$ is compact whenever one (and therefore all) of the following equivalent conditions is satisfied.

- Every open cover of $X$ contains a finite subcover;

- Every filter on $X$ has a cluster point;

- Every ultrafilter on $X$ is convergent.

We note that, in contrast to [5] or [14], Hausdorffness is not presupposed.

The following [17, Ch. 5, Theorem 6] is the Alexander Subbase Theorem:

AST. Let $\mathcal{S}$ be a subbase of $\mathcal{O}$. If any cover of $X$ by elements of $\mathcal{S}$ contains a finite subcover, then $X$ is compact.

We are interested in a more general form of AST in which the space $X$ is replaced by a filter base or, equivalently, a filter. We need to introduce some terminology. Let $\mathcal{P}, \mathcal{H}$ be families of subsets of $X$. We write $\mathcal{P} \# \mathcal{H}$ and say that $\mathcal{P}$ meshes with $\mathcal{H}$ if $P \cap H \neq \emptyset$ for each $P \in \mathcal{P}$ and each $H \in \mathcal{H}$. We say that $\mathcal{H}$ is a cover (resp. undercover) of a set $A \subset X$ if $A \subset \cup \mathcal{H}$ (resp. $A \subset \bigcup \overline{\mathcal{H}}=\bigcup\{\bar{H}: H \in \mathcal{H}\})$.

A filter in $\mathcal{O}$ is a nonempty subfamily $\mathcal{G} \subset \mathcal{O}$ which does not contain the empty set, is stable under finite intersections and such that if $G \in \mathcal{G}$ and $G \subset H \in \mathcal{O}$, then $H \in \mathcal{G}$. A filter which is a maximal element with respect

2000 Mathematics Subject Classification: Primary 54D30.

Key words and phrases: Alexander subbase theorem, Tikhonov product theorem, filter, ultrafilter, compact, midcompact, absolutely closed, USCO-map. 
to inclusion in the family of filters in $\mathcal{O}$ is called an ultrafilter in $\mathcal{O}$. Note that if $\mathcal{O}=2^{X}$ (i.e., the topology is discrete), we may drop "in $\mathcal{O}$ ", recovering the usual notion of an (ultra) filter in $X$.

A variant of compactness, called absolute closedness [2], [5] or H-closedness [3], [14], was defined in Hausdorff spaces. We do not presuppose Hausdorffness and because of our stress on the analogy with the compact case, it is convenient to switch to a more flexible term of midcompactness. The term was actually introduced as "midcompactoidness" in a survey of "compactoid filters" [18]. However, in recent papers there is a tendency to return to the classical terminology in which the term "compact filter" (instead of compactoid filter) is used. The use of "midcompactness" is especially convenient when dealing with many sorts of midcompact-like families of sets (see [11]).

We reserve $\mathcal{B}$ to denote a filter base in $X$.

Cover-Filter DuAlity. The following conditions are equivalent.

(1) (resp. (2)) For each open cover $\mathcal{P}$ of $A$, there exist $B \in \mathcal{B}$ and a finite subfamily $\mathcal{P}_{0} \subset \mathcal{P}$ covering (resp. undercovering) $B$.

$\left(1_{*}\right)$ (resp. $\left.\left(2_{*}\right)\right)$ For each filter (resp. filter in $\left.\mathcal{O}\right) \mathcal{F}$ such that $\mathcal{F} \# \mathcal{B}$, $\mathcal{F}$ has a cluster point in $A$.

$\left(1^{\prime}\right)$ (resp. $\left.\left(2^{\prime}\right)\right)$ For each ultrafilter (resp. ultrafilter in $\left.\mathcal{O}\right) \mathcal{U}$ such that $\mathcal{U} \# \mathcal{B}, \mathcal{U}$ converges to a point in $A$.

Proof. Only $\left(2^{\prime}\right) \Rightarrow\left(2_{*}\right)$ is not standard. Suppose $\mathcal{F}$ is a filter in $\mathcal{O}$ meshing with $\mathcal{B}$. Denote by $\mathcal{F} \vee \mathcal{B}$ the filter generated by the base $\{F \cap B: F \in \mathcal{F}$, $B \in \mathcal{B}$ \}. Let $\mathcal{U}$ be an ultrafilter finer than $\mathcal{F} \vee \mathcal{B}$. Define

$$
\mathcal{O}(\mathcal{U})=\{O \in \mathcal{O}: \exists U \in \mathcal{U} \text { such that } U \subset O\} .
$$

Then $\mathcal{O}(\mathcal{U})$ is an ultrafilter in $\mathcal{O}$ which is finer than $\mathcal{F}$ and meshes with $\mathcal{B}$. By $\left(2^{\prime}\right), \mathcal{O}(\mathcal{U})$ converges to a point $x \in A$. Clearly, $x$ is a cluster point of $\mathcal{F}$. This shows $(2 *)$.

We say that $\mathcal{B}$ is compact (resp. midcompact) at $A$ if any (and therefore all) of the respective conditions above is satisfied. We also consider (mid) compactness at a family of subsets of $X$. If $\mathcal{A}$ is such a family, then $\mathcal{B}$ is said to be (mid) compact at $\mathcal{A}$ if it is so at $A$, for each $A \in \mathcal{A}$. If $\mathcal{A}=\mathcal{B}$, then $\mathcal{B}$ is said to be selfcompact (resp. selfmidcompact).

Let $\mathcal{S}$ be a subbase of $\mathcal{O}$ and let $\mathbb{S}$ be the class of all covers of $A$ consisting of sets from $\mathcal{S}$. We say that $\mathcal{B}$ is $\mathbb{S}$-compact (resp. midcompact) at $A$ if for every $\mathcal{Q} \in \mathbb{S}$ there exist $B \in \mathcal{B}$ and a finite subfamily $\left\{Q_{1}, \ldots, Q_{n}\right\} \subset \mathcal{Q}$ covering (resp. undercovering) $B$.

AST-General Form. Let $\mathcal{B}$ be $\mathbb{S}$-compact (resp. midcompact) at $A$. Then $\mathcal{B}$ is compact (resp. midcompact) at $A$. 
Proof. Denying $\left(1^{\prime}\right)$ (resp. $\left(2^{\prime}\right)$ ), let $\mathcal{U}$ be an ultrafilter (resp. in $\left.\mathcal{O}\right)$, meshing with $\mathcal{B}$ and without a limit point in $A$. We claim that:

For each $x \in A$, there exists $G(x) \ni x, G(x)$ in $\mathcal{S}$, such that $G(x)$ does not mesh with $\mathcal{U}$.

Otherwise, there exists $x$ such that the sets $G(x) \in \mathcal{S}$ containing $x$ mesh with $\mathcal{U}$. As $\mathcal{U}$ is an ultrafilter (resp. in $\mathcal{O}$ ) and $G(x)$ are open, the sets $G(x)$ would belong to $\mathcal{U}$. As finite intersections of such sets form a base of the neighborhood filter of $x$, the point $x$ would be a limit point of $\mathcal{U}$.

Applying the assumption of the theorem to the cover $\{G(x): x \in A\}$, we find $x_{1}, \ldots, x_{k}$ and $B \in \mathcal{B}$ such that $B \subset \bigcup_{i=1}^{k} G\left(x_{i}\right)$ (resp. $\left.B \subset \bigcup_{i=1}^{k} \overline{G\left(x_{i}\right)}\right)$.

On the other hand, as $G\left(x_{i}\right)$ 's do not mesh with $\mathcal{U}$, it follows that neither does $G=\bigcup_{i=1}^{n} G\left(x_{i}\right)$. This gives a contradiction in the compact case, because $B \subset G$ and $\mathcal{B} \# \mathcal{U}$. In the "mid case", $U \in \mathcal{U}$ being open, $U \cap \bar{G}=\emptyset$ for some $U \in \mathcal{U}$. This contradicts the fact that $\bar{G} \supset B \in \mathcal{B}$ and $\mathcal{U} \# \mathcal{B}$.

Corollary 1 . If $\mathcal{B}$ is $\mathbb{S}$-selfcompact (resp. $\mathbb{S}$-selfmidcompact), then it is selfcompact (resp. selfmidcompact).

The corollary solves a question left open in [18] (see the comment after Th. 5.3 there). Actually, the statement in parentheses seems to be new even in the special case of an absolutely closed space $X$. We now deduce a general Tikhonov Product Theorem (TPT):

TPT-General Form. Let $X_{\iota}$ be a filter on $X_{\iota}$, which is compact (resp. midcompact) at $A_{\iota}$. The product filter $\mathcal{X}=\prod_{\iota \in I} X_{\iota}$ on $X=\prod_{\iota \in I} X_{\iota}$ is compact (resp. midcompact) at $A=\prod_{\iota \in I} A_{\iota}$.

Proof. Let $\mathcal{S}$ be a subbase of $X$ defined by the sets $\operatorname{pr}_{\iota}^{-1}\left(E_{\iota}\right), \iota \in I$, where $E_{\iota}$ is an open set in $X_{\iota}$. Suppose that $\mathcal{G}$ is a family of sets from $\mathcal{S}$ so that there is no $D \in \mathcal{X}$ and no finite subfamily of $\mathcal{G}$ covering (resp. undercovering) $D$. Let $\mathcal{E}_{\iota}$ be the family of all open sets in $X_{\iota}$ such that $\operatorname{pr}_{\iota}^{-1}\left(\mathcal{E}_{\iota}\right) \subset \mathcal{G}$. As $\operatorname{pr}_{\iota}(X)=X_{\iota}$, there is no $B_{\iota} \in X_{\iota}$ and no finite subfamily of $\mathcal{E}_{\iota}$ which covers (resp. undercovers) $B_{\iota}$. By compactness (resp. midcompactness) of $X_{\iota}$ at $A_{\iota}$, there is $a_{\iota} \in A_{\iota}$ not covered by $\mathcal{E}_{\iota}$. Then $a=\left(a_{\iota}\right) \in A$ is not covered by $\mathcal{G}$. Hence $\mathcal{X}$ is $\mathbb{S}$-compact at $A$. Now apply the general form of AST.

The "compact case" of the theorem goes back to Pettis [19] (who thought in terms of nets). The "midcompact case" has been announced in [12] but no proof has ever been published. In the special case of the product of spaces this is a theorem of Chevalley and Frink [9].

Let us consider a still more general compactness theorem [13]. Recall that a USCO-map is an upper semicontinuous function taking nonempty compact values. 
Corollary 2. For each $\iota \in I$, let $\Gamma_{\iota}: X_{\iota} \rightrightarrows Y_{\iota}$ be a USCO-map. Then the product map

$$
\Gamma: \prod_{\iota \in I} X_{\iota} \rightrightarrows Y=\prod_{\iota \in I} Y_{\iota}
$$

defined by $\Gamma\left(\left(x_{\iota}\right)_{\iota \in I}\right)=\prod_{\iota \in I} \Gamma_{\iota}\left(x_{\iota}\right)$ is also a USCO-map.

The following proof shows that the filter generalizations may be useful. Indeed, let $\mathcal{N}_{\iota}=\mathcal{N}\left(x_{\iota}\right)$ be the neighborhood filter of $x_{\iota}$. Then the image filter $\Gamma_{\iota}\left(\mathcal{N}_{\iota}\right)$ is compact at $\Gamma_{\iota}\left(x_{\iota}\right)$. Hence the product filter $\prod_{\iota \in I} \Gamma_{\iota}\left(\mathcal{N}_{\iota}\right)$ is compact at $\prod_{\iota \in I} \Gamma\left(x_{\iota}\right)$. This also means that

$$
\left(\prod_{\iota \in I} \Gamma_{\iota}\right)\left(\prod_{\iota \in I} \mathcal{N}_{\iota}\right)=\Gamma(\mathcal{N}(x))
$$

is compact at $\Gamma(x)$, where $x=\left(x_{\iota}\right)_{\iota \in I}$, or, which is the same, that $\Gamma$ is upper semicontinuous at $x$.

Remark 1. The Alexander Subbase Theorem is [1, Theorem 1]. As its first corollary [1, Theorem 2], Alexander gives the Tikhonov Product Theorem declaring its proof evident. Kelley gives a proof in [17]. Nonetheless Kelley, discussing the connections with the axiom of choice in [16], speaks about "Alexander's proof" and calls it "the most illuminating" of all the proofs (of TPT). The present paper adapts the "Alexander approach" to the more general situation studied here.

Kelley also gives in [17] a second proof calling it "Bourbaki's proof". Actually, the proof is the one of Chevalley and Frink [9]. A proof $a$ la Bourbaki [5] of the general TPT as stated above will appear in [10]. Whether it is less "illuminating" than Alexander's proof is perhaps a matter of taste. It is undeniable though that Bourbaki's proof works in non-topological situations of convergence theory (see for instance [4]).

REMARK 2. In [6], a book aimed at beginning students, Buskes and van Rooij have a section entitled "Dates from the History of General Topology" in which the important events are listed. Here is one of the entries:

- 1937 Cech proves the Tychonoff Theorem.

I tried to trace a source of this "canard" in the literature. The earliest claim of this type that I found, was in [20]. Walter Rudin apparently liked to stir calm waters; some other historical remarks in Appendix B of the book prompted further, rather sceptical, comments in the Russian translation of [20] (Moscow 1975). In the case at hand, Rudin briefly summarizes the contents of [21] and [8] concluding that "it appears that Cech proved the Tychonoff theorem, whereas Tychonoff found the Čech compactificationa good illustration of the historical reliability of mathematical nomenclature". 
The claims were later examined again by Cameron in [7]. Although the conclusion about the compactification is, to some extent, acknowledged in [8] by Čech himself, both Cameron and later Simon in [15] find the Čech-Stone or Stone-Čech denominations to be correct. On the other hand, similarly to Rudin, Cameron concludes that the full version of the Tikhonov theorem is due to Čech. Yet, while it is true that in [21] only products of unit intervals are considered and in [8] one finds the TPT for topological spaces, it is also true that Tikhonov states the general theorem in [22]. Besides, this last information can also be found in [14]. To conclude, the Tikhonov theorem is due - no sensation here- to Tikhonov.

\section{References}

[1] J. W. Alexander, Ordered sets, complexes and the problem of compactification, Proc. Nat. Acad. Sci. USA 25 (1939), 296-298.

[2] P. Alexandroff et P. Urysohn, Mémoire sur les espaces topologiques compacts, Verh. Kon. Akad. Wetensch. Amsterdam 14 (1929), 1-96.

[3] P. Alexandroff und H. Hopf, Topologie, Chelsea, New York, 1965 (reprint of the original 1935 edition).

[4] R. Beattie and H. P. Butzmann, Convergence Structures and Applications to Functional Analysis, Kluwer, 2002.

[5] N. Bourbaki, Topologie générale, Ch. I, II, 3-me éd., Hermann, Paris, 1961.

[6] G. Buskes and A. van Rooij, Topological Spaces, Springer, 1997.

[7] D. E. Cameron, The birth of the Stone-Čech compactification, in: Rings of Continuous Functions, Lecture Notes in Pure and Appl. Math. 95, Dekker, New York, 1985, $67-78$.

[8] E. Čech, On bicompact spaces, Ann. of Math. 38 (1937), 823-844.

[9] C. Chevalley and O. Frink, Bicompactness of Cartesian products, Bull. Amer. Math. Soc. 47 (1941), 612-614.

[10] B. L. Davis and I. Labuda, Infinite products of filters, Math. Slovaca, to appear.

[11] - - - Unity of compactness, preprint, 2005, submitted.

[12] S. Dolecki et G. H. Greco, Familles pseudotopologiques de filtres et compacité, C. R. Acad. Sci. Paris 296 (1983), 211-214.

[13] L. Drewnowski and I. Labuda, On minimal upper semicontinuous compact-valued maps, Rocky Mountain J. Math. 20 (1990), 737-752.

[14] R. Engelking, General Topology, PWN-Polish Sci. Publ., Warszawa, 1977.

[15] M. Katětov and P. Simon (eds.), The Mathematical Legacy of Eduard Čech, Birkhäuser, 1993.

[16] J. L. Kelley, The Tychonoff product theorem implies the axiom of choice, Fund. Math. 37 (1950), 75-76.

[17] —, General Topology, Van Nostrand, 1955.

[18] I. Labuda, Compactoidness, Rocky Mountain J. Math. 36 (2006), 555-574.

[19] B. J. Pettis, Cluster sets of nets, Proc. Amer. Math. Soc. 22 (1969), 386-391.

[20] W. Rudin, Functional Analysis, McGraw-Hill, 1973.

[21] A. Tychonoff, Über topologische Erweiterung von Raümen, Math. Ann. 102 (1930), $544-561$. 
[22] A. Tychonoff, Ein Fixpunktsatz, ibid. 111 (1935), 767-776.

Iwo Labuda

Department of Mathematics

University of Mississippi

University, MS 38677, U.S.A.

E-mail: mmlabuda@olemiss.edu

Received January 15, 2006;

received in final form September 3, 2006 